\title{
Distributed smart charging of electric vehicles for balancing wind energy
}

\author{
Kevin Mets, Filip De Turck, Chris Develder \\ Dept. of Information Technology - IBCN - Ghent University - IBBT \\ G. Crommenlaan 8 Bus 201, 9050 Ghent, Belgium \\ Email: Kevin.Mets@intec.ugent.be
}

\begin{abstract}
To meet worldwide goals of reducing $\mathrm{CO}_{2}$ footprint, electricity production increasingly is stemming from so-called renewable sources. To cater for their volatile behavior, so-called demand response algorithms are required. In this paper, we focus particularly on how charging electrical vehicles (EV) can be coordinated to maximize green energy consumption. We present a distributed algorithm that minimizes imbalance costs, and the disutility experienced by consumers. Our approach is very much practical, as it respects privacy, while still obtaining near-optimal solutions, by limiting the information exchanged: i.e. consumers do not share their preferences, deadlines, etc. Coordination is achieved through the exchange of virtual prices associated with energy consumption at certain times.

We evaluate our approach in a case study comprising 100 electric vehicles over the course of 4 weeks, where renewable energy is supplied by a small scale wind turbine. Simulation results show that $68 \%$ of energy demand can be supplied by wind energy using our distributed algorithm, compared to $73 \%$ in a theoretical optimum scenario, and only $40 \%$ in an uncoordinated business-as-usual (BAU) scenario. Also, the increased usage of renewable energy sources, i.e. wind power, results in a $45 \%$ reduction of $\mathrm{CO}_{2}$ emissions, using our distributed algorithm.
\end{abstract}

\section{INTRODUCTION}

Worldwide energy supply will increasingly depend on (distributed) renewable energy sources, stimulated by initiatives such as the EU "20-20-20" targets [1] that aim to increase the share of renewable energy sources, and reduce energy consumption and emission of greenhouse gasses. The power grid was however not designed to support such local production and the bidirectional energy flows it introduce. Especially the intermittent nature of these energy sources is problematic, as energy supply and demand have to be matched in a power grid. Changing demand profiles, for example due to the introduction of electric vehicles, only aggravate the problem. However, demand side management (DSM) methods are proposed to deal with such challenges, by modifying consumer demand patterns.

In this work, we focus on a distributed DSM algorithm for balancing supply from renewable energy sources, and demand from charging electric vehicles. Our approach is inspired on iterative or negotiated pricing algorithms and is quite elegant, in that it respects privacy concerns (consumers do not share any behavioral information) while allowing near-optimal demand supply matching. We discuss related work and summarize our contribution in Section II. Section III summarizes our problem statement. The design of the distributed algorithm is discussed in Section IV. We present a case study and simulation results in Section V and Section VI and conclude in Section VII.

\section{RELATED WORK}

We start our overview of related work by discussing several distributed algorithms based on (virtual) energy prices that are negotiated in an iterative fashion, as these are closely related to the proposed approach. A real-time pricing algorithm based on utility maximization for a smart grid consisting of an energy provider and multiple subscribers is presented in [2]. Subscribers and the energy provider run a distributed algorithm based on dual decomposition that calculates: (i) optimal energy consumption level for each subscriber, (ii) optimal generating capacity for the energy provider, and (iii) the optimal price values to be advertised by the energy provider. The algorithm presented in [2] is extended to account for uncertainty on the demand (e.g. stemming from communication issues, measurement errors and deviating behavior) [3]. Both approaches use utility functions and consumption intervals to define the flexibility of subscribers. However, this model does not account for specific deadlines that certain loads may have, e.g. electrical vehicles (EVs). A similar algorithm is presented in [4] that includes multiple consumer types, including EVs, whereas [2], [3] only consider one type of flexible consumer.

The exchange of control messages between the different components of the system is essential for the aforementioned coordination methods. Therefore, the communication network should be designed appropriately. Communication limitations are studied in [5], and the impact of lost messages is studied in [4], [5]. Both studies have demonstrated that the distributed algorithms can deal with lost messages, however, time needed to obtain a solution is increased.

Note that the aforementioned methods do not specifically consider renewable energy sources or target the coordinated charging of electric vehicles. However, alternative methods exist that do focus on these topics. A multi-agent system and two possible scheduling strategies, reactive and proactive, are proposed in [6] to reduce imbalance costs. A key concept in the proposed approaches are so called intention graphs, which represent the flexibility of a fleet of EVs. Both strategies are evaluated in a scenario with distributed generation from PV installations. The proactive strategy outperforms the reactive strategy ( $44 \%$ vs. $14 \%$ cost reduction) due to spreading the imbalance risk over time, thereby avoiding large concentrated imbalances. Another approach is presented in [7]: charging start times depend on a single $\mathrm{EV}$ connection rate that determines on average how many EVs can start charging per time unit. Determination of this connection rate is based on 
concepts from queueing theory and statistical analysis. The main benefit of this approach is the minimal communication network requirements: only a single number, the connection rate, is broadcast to control the starting times.

The main contribution of our current paper is the adaptation and application of a DSM approach similar to [3]-[5], but focusing on balancing wind energy supply and electric vehicle charging demand. Summarized, our contributions are as follows:

- We extended the model to cater for supply-demand matching involving volatile energy sources (rather than minimizing generation costs of classical sources).

- We perform a realistic case study, and compare the approach to a business-as-usual scenario and a best-case scenario.

- We focus on evaluating the potential of a specific type of subscribers, i.e. electric vehicles, to balance supply and demand.

- We analyze the performance of our coordination mechanism in terms of renewable energy consumption versus non-renewable, and express the environmental benefit in $\mathrm{CO}_{2}$ emission reduction.

\section{PROBLEM STATEMENT: Wind BALANCING}

Integration of renewable energy sources, i.e. wind energy, in the power grid is challenging due to the intermittent nature of these energy sources. Therefore, we propose a distributed algorithm to balance renewable energy from wind generators with the charging demand of electric vehicles, thereby increasing renewable energy consumption, and reducing emissions of greenhouse gasses. We approach this problem from the viewpoint of a balance responsible party (BRP), also known as access responsible party (ARP), that is responsible to ensure that energy supply matches energy consumption during a given time period: if the balance is not maintained, the BRP is required to pay imbalance costs. Therefore, the objective of the BRP is to minimize the imbalance costs. Nevertheless, the wishes and preferences of subscribers have to be respected, and are therefore accounted for in our approach, while maintaining privacy.

\section{DISTRIBUTED SMART CHARGING}

We consider a power system that consists of a BRP, multiple subscribers, and a coordinator. Subscribers are flexible energy consumers (which in our use case will be electric vehicle owners), that are willing to change their demand patterns in order to aid in maintaining balance between supply and demand (and hence will receive some incentives to do so). The coordinator is responsible to coordinate the actions of the BRP and subscribers. Figure 1 gives a system overview. Subscribers and the BRP only interact with the coordinator, exchanging virtual prices or consumption/production schedules.

A distributed approach was chosen to improve the scalability of the algorithm, to support large groups of subscribers. (To further scale up, we can deploy also multiple coordinators that independently manage their own set of subscribers.) Subscribers determine independently a consumption schedule, and scalability can be further enhanced using a hierarchical

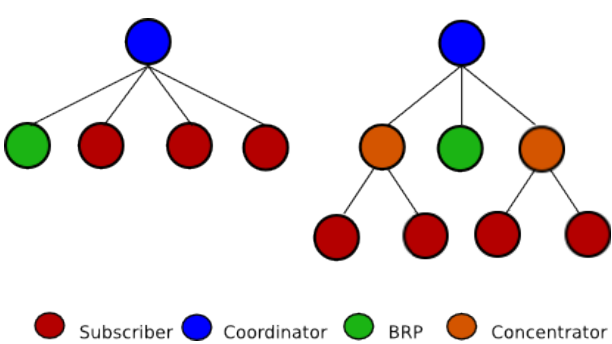

Fig. 1. System overview.

architecture, in which e.g. data concentrators are used, as illustrated in Fig. 1. The privacy of the subscribers is ensured, as only limited information, i.e. the planned energy consumption schedule and virtual energy prices, are exchanged. Smart meters measure the energy consumption of subscriber regularly, e.g. every 15 minutes. Therefore, the information exchanged does not imply additional privacy issues, as it is already collected for billing purposes. Note that we use the term virtual energy prices to indicate that they are used as a control signal, rather than real costs to be paid by the consumer. Other parameters, such as arrival and departure times, vehicle properties, and the willingness to participate in balancing demand and supply, are not exchanged.

\section{A. Conceptual view}

In this section we will introduce the iterative distributed algorithm from a high level perspective. Sections IV-B, IV-C, and IV-D will provide more details regarding the mathematical framework behind the distributed algorithm.

We assume that time is divided in time slots, e.g. of 15 minutes. Wind power generation forecasts are made available at the start of each time slot $t$ for the next $T$ consecutive time slots. At the beginning of each time slot $t$ the distributed algorithm is executed. During the execution of the distributed algorithm, the BRP and subscribers determine supply and demand schedules. The iterative distributed algorithm consists of the following steps:

1) Initialization:

a) The BRP updates the wind power generation forecast $w=\left[w_{1}, w_{2}, \ldots, w_{T}\right]$ for the next $T$ time slots.

b) The coordinator initializes a (virtual) price vector $p=$ $\left[p_{1}, p_{2}, \ldots, p_{T}\right]$ used to steer demand and supply.

2) The coordinator sends the price vector $p$ to the subscribers and the BRP.

3) Each subscriber calculates an energy consumption schedule based on the prices and its requirements and preferences, and sends the schedule back to the coordinator.

4) The BRP determines an energy production schedule based on the wind power generation forecast and the prices, and sends it back to the coordinator.

5) The coordinator collects the consumption schedules from the subscribers and production schedules from the BRP.

6) The coordinator compares the received consumption and production schedules.

a) If the discrepancy between supply and demand is below a predefined acceptance level, or the maximum 
number of iterations is reached, the algorithm terminates and the subscribers and BRP are notified that the schedules are final.

b) Otherwise, the coordinator updates the price vector $p$ and a new iteration is started from step 2 .

The distributed algorithm is executed at the beginning of each time slot to incorporate new information from updated wind power generation forecasts, and to deal with subscribers that connected or disconnected, or changes in subscriber requirements. The iterative design of the algorithm allows us to maintain privacy in the system.

We will now discuss the mathematical framework.

\section{B. Subscribers}

Subscribers are flexible energy consumers that are willing to change their energy demand patterns to assist in maintaining a balance between supply and demand. In this work, we focus on electric vehicles, as they represent a significant but also flexible load to the grid. Since the main concern of electric vehicle owners is to obtain a specified amount of energy $E_{k}$ within a certain time frame, charging of electric vehicles can be adjusted (slower/faster) and shifted in time to coincide with supply from renewable energy sources.

We assume there are $K$ subscribers identified by $k \in$ $\{1,2, \ldots, K\}$. The coordination mechanism will decide on a schedule for $T$ consecutive time slots: a subscriber will plan a consumption schedule $x^{k}=\left[x_{1}^{k}, x_{2}^{k}, \ldots, x_{T}^{k}\right]$. Our subscribers, being EVs, will have a deadline $T_{k}$ by which the EV needs to be charged. The total energy to be acquired by that time will be denoted as $E_{k}$. For now, we assume $T_{k} \leq T$. Constraints (1) and (2) specify that subscriber $k$ can only consume energy between the start of the scheduling window and the departure time slot. Energy consumption is limited by $p_{k, \max }$, e.g. based on the capabilities of the charging station.

$$
\begin{aligned}
0 \leq x_{t}^{k} \leq p_{k, \max } & , \quad \forall t \in\left\{1, \ldots, T_{k}-1\right\} \\
x_{t}^{k}=0 & , \quad \forall t \in\left\{T_{k}, \ldots, T\right\}
\end{aligned}
$$

Constraint (3) specifies that subscriber $k$ needs to obtain a specified amount of energy $E_{k}$ before time slot $T_{k} \leq T$, the departure time of the subscriber.

$$
\sum_{t=1}^{T_{k}} x_{t}^{k}=E_{k}
$$

Instead of only modeling the energy requirements of the consumer, we also want to enable the user to indicate their willingness to participate. Therefore, willingness of each subscriber to participate in balancing supply and demand is modeled with a disutility function. The disutility function (4) represents the discomfort associated with deviating from the preferred charging power $p^{k}$ of subscriber $k$.

$$
D_{t}^{k}\left(x_{t}^{k}\right)=\beta_{k}^{t} \cdot\left(p^{k}-x_{t}^{k}\right)^{2}
$$

An example of the preferred charging power $p^{k}$ is the maximum charging power supported by the charging station or EV. Ideally, this preferred charging power is obtained automatically from the charging station or EV, otherwise the user is required to provide such information. The disutility function employs a private parameter $\beta_{k}^{t}$ to influence the willingness of subscriber $k$ to participate in balancing supply and demand during time slot $t$. Subscribers can choose a constant value for $\beta_{k}^{t}$ to indicate no preferences in time, or subscribers can choose a value that changes over time, for example to indicate a preference for being charged sooner rather than later. Note that the specific design of the disutility function requires further research in order to present the user with parameter choices that are simpler and more intuitive. However, the presented disutility function results in the expected behavior, i.e. supply and aggregated demand are matched as best as possible, and enables the distributed algorithm presented in section IV-E.

\section{Balance Responsible Party}

The balance responsible party (BRP) is responsible to maintain balance between supply and demand within his balancing zone. When this balance is not maintained, an imbalance cost is incurred to the BRP. Therefore, the BRP aims to keep the imbalance as small as possible over time. We consider a scenario where the balancing zone of the BRP consists of a wind turbine (park) and subscribers. The BRP is provided a forecast of energy generation from a wind turbine (park), and aims to balance energy demand from the subscribers with the forecast of the supply from the wind turbine (park).

We model the imbalance through a convex cost function. Since we assume negative imbalance (supply $<$ demand) is equally bad as positive imbalance (supply $>$ demand), we use a quadratic function (5). (Note that [2] rather used a strictly increasing one, given its different objective.)

$$
C_{t}\left(d_{t}\right)=\alpha \cdot\left(w_{t}-d_{t}\right)^{2}
$$

In (5), we define $d_{t}$ as the aggregated power demand of all subscribers during time slot $t$, and $w_{t}$ as the forecast of wind energy supply during time slot $t$. It is clear that when supply and demand are in balance, no imbalance costs are incurred to the BRP. We add constraint that specifies that demand $d_{t}$ is limited below a certain upper bound $d_{\max }$, reflecting the limited grid capacity..

$$
d_{t}<d_{\max }
$$

We have selected this quadratic (virtual) cost function based on technical and economical motivations. On a technical level, we prefer that if deviations occur, that they are spread in time, similar to the proactive scheduling strategy proposed in [6]. On an economical level, we were inspired by the cost of large deviations, which is based on a quadratic function of the imbalance, as defined by the Belgian transmission system operator (TSO) Elia [8].

\section{Social Welfare}

We aim to minimize the sum of imbalance costs $C_{t}(\cdot)$ incurred to the BRP, and the disutility $D_{t}^{k}(\cdot)$ of the subscribers that aid in balancing. This can also be interpreted as an aim to maximize social welfare. We define $x_{t}^{k}$ as the energy demand of subscriber $k$ during time slot $t$. Energy supplied by the BRP during time slot $t$ must match the energy demand from the subscribers. Therefore, the global optimization objective 
that we wish to minimize is (7). We assume constraints (1), (2), (3), and (6).

$$
\min _{x_{t}^{k}}\left\{\sum_{t=1}^{T} C_{t}\left(\sum_{k=1}^{K} x_{t}^{k}\right)+\sum_{k=1}^{K} \sum_{t=1}^{T} D_{t}^{k}\left(x_{t}^{k}\right)\right\}
$$

Due to the selection of the cost and utility functions, we are dealing with a convex optimization problem. However, in order to solve this optimization problem centrally, the disutility function and private parameters of each subscriber must be known, which gives rise to privacy issues. Also, solving such optimization problems centrally is not scalable for large numbers of subscribers. Therefore, we aim to solve this problem in a distributed way. Ideally, the BRP and each subscriber should determine their energy production or consumption schedule independently, but this is complicated by the dependency between the energy consumption schedule from the subscribers, and the associated imbalance costs incurred to the BRP.

\section{E. Distributed algorithm}

To solve the convex optimization problem in a distributed way, we apply dual decomposition: the optimization problem is decomposed into multiple smaller sub problems that can be solved independently. We reformulate the optimization problem in (8) and introduce new variables $d_{t}$ defined in constraint (9) as the aggregated demand of all subscribers during time slot $t$. We assume constraints (1), (2), (3), and (6).

$$
\begin{gathered}
\min _{d_{t}, x_{t}}\left\{\sum_{t=1}^{T} C\left(d_{t}\right)+\sum_{k=1}^{K} \sum_{t=1}^{T} D_{t}^{k}\left(x_{t}^{k}\right)\right\} \\
\sum_{k=1}^{K} x_{t}^{k}=d_{t} \quad, \quad \forall t \in\{1,2, . ., T\}
\end{gathered}
$$

We apply the method of Lagrange multipliers to move the coupling constraints (9) to the objective function and obtain the following objective function.

$$
\sum_{t=1}^{T} C\left(d_{t}\right)+\sum_{k=1}^{K} \sum_{t=1}^{T}\left(D_{t}^{k}\left(x_{t}^{k}\right)+\lambda_{t}\left(x_{k}^{t}-d_{t}\right)\right)
$$

The Lagrange multipliers $\lambda_{t}$ can be interpreted as virtual prices, used to manage the amount of resources used. Next, we rearrange the terms of the objective function, and it becomes clear that the objective function is separable, and therefore eligible for decomposition methods, such as dual decomposition, that can be solved in a distributed fashion using the subgradient method.

$$
\sum_{t=1}^{T}\left(C\left(d_{t}\right)-\lambda_{t} d_{t}\right)+\sum_{k=1}^{K} \sum_{t=1}^{T}\left(D_{t}^{k}\left(x_{t}^{k}\right)+\lambda_{t} x_{t}^{k}\right)
$$

We can decompose this problem in $K+1$ sub-problems that can be solved in parallel. However, we will require multiple iterations during which the $K+1$ sub-problems are solved, in order to solve the global optimization problem (7). In each iteration, a new vector $\lambda$ will be used, based on the results of the previous iterations. We indicate an iteration by $i$. Then, given a vector $\lambda^{i}=\left[\lambda_{1}^{i}, \lambda_{2}^{i}, \ldots, \lambda_{T}^{i}\right]$, each subscriber will solve sub-problem (12), assuming constraints (1), (2), and (3).

$$
\min _{x_{t}^{k, i}}\left\{\sum_{t=1}^{T}\left(D_{t}^{k}\left(x_{t}^{k, i}\right)+\lambda_{t}^{i} x_{t}^{k, i}\right)\right\}
$$

Given the same vector $\lambda^{i}=\left[\lambda_{1}^{i}, \lambda_{2}^{i}, \ldots, \lambda_{T}^{i}\right]$ as the subscribers, the BRP will solve sub-problem (13), assuming constraints (6).

$$
\min _{d_{t}^{i}}\left\{\sum_{t=1}^{T}\left(C\left(d_{t}^{i}\right)-\lambda_{t}^{i} d_{t}^{i}\right)\right\}
$$

Energy consumption vectors $x^{k, i}=\left[x_{1}^{k, i}, x_{2}^{k, i}, \ldots, x_{T}^{k, i}\right]$ are collected from each subscriber, and an energy production vector $d^{i}=\left[d_{1}^{i}, d_{2}^{i}, \ldots, d_{T}^{i}\right]$ is collected from the balance responsible party, and a new price vector $\lambda^{i+1}$ will be calculated according to (14) in which $\gamma$ represents the step size (to be chosen sufficiently small).

$$
\lambda_{t}^{i+1}=\lambda_{t}^{i}+\gamma \cdot\left[\sum_{k=1}^{K} x_{t}^{k, i}-d_{t}^{i}\right]
$$

The selection of the step size rule is important for the convergence of the subgradient algorithm [9]. For nondifferentiable objective functions, the subgradient algorithm is guaranteed to converge to a near optimal solution as a result of the constant step size rule used. For other step size rules, such as the diminishing step size rule, the subgradient algorithm is guaranteed to converge to the optimal value. However, for differentiable objective functions, such as in this case, the subgradient method converges to the optimal solution, provided the chosen step size is small enough [9]. Therefore, we selected the constant step size rule for simplicity, i.e. to reduce the number of parameters that have to be configured.

Note that due to our choice in imbalance cost function (5) and constraint (9), the virtual prices can become negative, otherwise supply and demand will not necessarily match.

Each subscriber and the BRP will solve respectively subproblem (12) and (13) using the vector $\lambda^{i+1}$ to obtain energy consumption schedules $x_{k}^{i+1}$ and an energy production schedule $d_{k}^{i+1}$. This process is repeated until demand and supply converge, or another convergence criterion is reached (e.g. a maximum number of iterations). However, if a maximum number of iterations is used, convergence is not guaranteed.

\section{Case Study}

A time period of 4 weeks is simulated, divided in time slots of 15 minutes. A forecast and planning window of 24 hours or $T=96$ time slots is assumed. For each time slot, the distributed algorithm is executed, to consider new wind forecast data and new arrivals. We assumed a small wind turbine with peak power output of $30 \mathrm{~kW}$, supplying approximately the energy required for charging the electric vehicles. We simulated a set of 100 electric vehicles of which the arrivals, departures and energy requirements are derived from a statistical model of real-life vehicle usage data [10]. The battery capacity of each vehicle is $10 \mathrm{kWh}$ and the 
maximum power demand of each battery charger is set to 3.68 $\mathrm{kW}$. We evaluate the performance of the distributed algorithm discussed in section IV, and compare it to the performance of two other charging strategies, that correspond to a businessas-usual or worst case scenario without coordinated charging, and a best-case scenario. This way, we obtained lower and upper bounds of the performance of the distributed algorithm.

\section{A. Uncontrolled charging}

We consider a business-as-usual scenario in which electric vehicles are charged uncontrolled. As soon as a vehicle arrives at the charging station, charging is started and continues until the battery is fully charged, using the maximum charging rate.

\section{B. Distributed smart charging}

The distributed algorithm discussed in section IV is implemented in Java using CPLEX. We used and $\alpha=0.1$ in (5) and $\beta_{k}=0.1$ in (4).

\section{Optimal Benchmark}

We implemented an optimal benchmark or best-case scenario using quadratic programming, without considering the preferences of the subscribers (4) and BRP (5). Nevertheless, we do respect the energy requirements (3) from the subscribers, and our goal remains to balance supply and demand. We assume all arrivals, departures, and energy requirements for the entire duration of the experiment to be known beforehand, in order to calculate the benchmark metric. Then, assuming a wind generation schedule $w=\left[w_{1}, w_{2}, \ldots, w_{S}\right]$ for the entire experiment duration of $S$ time slots, optimal energy consumption schedules $x^{k}=\left[x_{1}^{k}, x_{2}^{k}, \ldots, x_{S}^{k}\right]$ are calculated for each subscriber $k \in\{1,2, \ldots, K\}$, that minimize the following performance metric:

$$
\sum_{t=1}^{S}\left(w_{t}-\sum_{k=1}^{K} x_{t}^{k}\right)^{2}
$$

Clearly, these assumptions are unrealistic, as drivers should be able to predict their energy requirements and time constraints weeks in advance, which is unlikely. However, with this assumed all-knowing strategy we obtain the most optimal results, and use it as a reference to evaluate other algorithms.

\section{RESUlTS AND DISCUSSION}

We give an overview of the case study results obtained for the different charging strategies. Fig. 2 gives an overview of the supply and demand curves for the different charging strategies over the course of 7 days. In the uncontrolled scenario, we clearly observe the peak demands in the evening when EV users arrive at home. Obviously, this does not coincide with peaks in wind production. The benchmark scenario and the scenario using the distributed algorithm follow supply from wind generation more closely, where the all-knowing benchmark as expected does a slightly better job (e.g. period from day 1 18:00 to day 2 12:00). Nonetheless, we note that the difference in performance seems limited, as we assess in the following subsections in terms of \% green energy usage and reduction of $\mathrm{CO}_{2}$ emission.

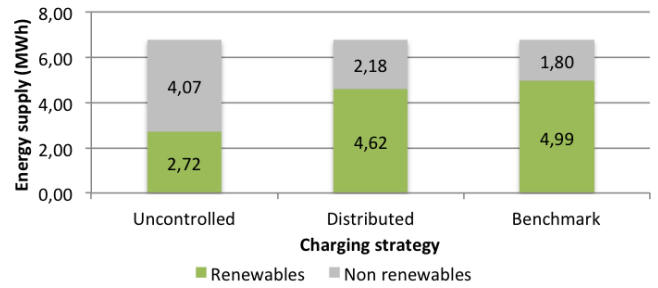

Fig. 3. Energy supply mix for the different charging strategies.

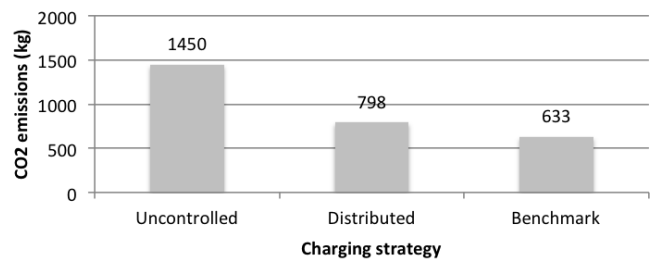

Fig. 4. $\mathrm{CO}_{2}$ emissions associated with the different charging strategies.

\section{A. Renewable Energy}

In this section we evaluate the impact of different charging strategies on the usage of renewable energy. The goal of the distributed algorithm is to balance wind energy supply and electric vehicle charging demand as much as possible, thus maximally use the available wind energy rather than from polluting sources. We consider the energy supply mix used for charging, which is summarized in Fig. 3.

Regardless of the charging approach, we require 6.79 MWh in total, for providing all vehicles with their required energy over the simulated time period. In the uncontrolled scenario, $2.72 \mathrm{MWh}$ or $40 \%$ of demand is supplied by wind energy. The best case scenario, which is considered in the benchmark, results in $4.99 \mathrm{MWh}$ or $73 \%$ of demand being supplied by wind energy. The performance of the distributed algorithm is close to that of the theoretical benchmark, and results in 4.62 MWh or $68 \%$ being supplied by wind energy. These results clearly indicate, that the distributed algorithm results in a substantial increase in the use of wind energy, despite the fact that only a limited amount of information is shared (cf. privacy concerns) between the components.

\section{B. $\mathrm{CO}_{2}$ Emissions}

The focus of the work presented in this paper is to balance demand from charging electric vehicles with supply from renewable energy sources, i.e. wind generators. However, due to the intermittent nature of these energy sources, conventional power generation is still required to provide additional reserve capacity, such as gas turbines or coal plants, which have higher $\mathrm{CO}_{2}$ emissions. By shifting energy consumption to times at which renewable energy is available, we reduce $\mathrm{CO}_{2}$ emissions associated with charging electric vehicles. Below, we quantitatively assess this reduction.

We assume that the carbon dioxide life cycle emissions for wind generation equal $7.4 \mathrm{~g} \mathrm{CO}_{2}-\mathrm{eq} / \mathrm{kWh}$, and nonrenewables emissions amount to $351 \mathrm{~g} \mathrm{CO}_{2} / \mathrm{kWh}$, based on the EU figures from [11]. (Note that $\mathrm{CO}_{2}$-eq takes into 


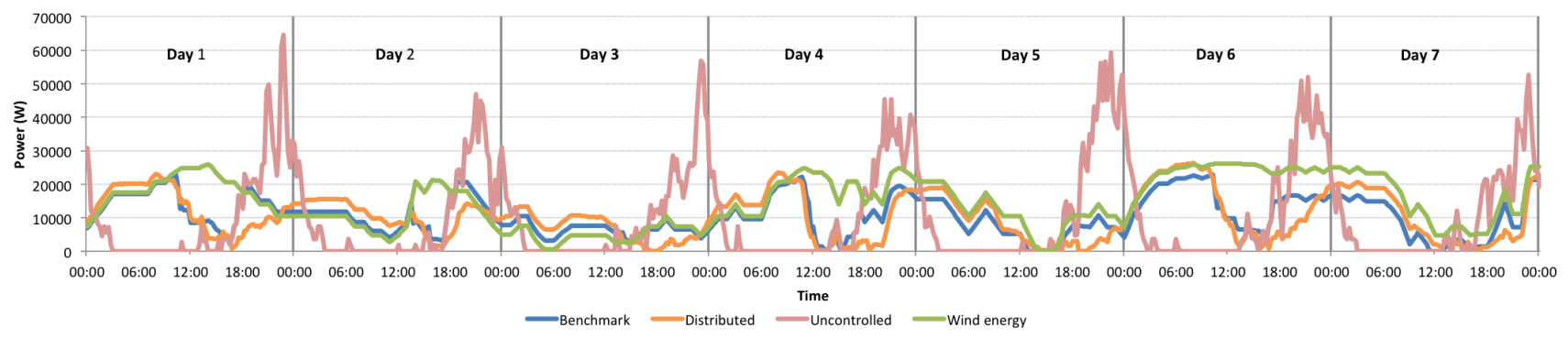

Fig. 2. Supply and demand curves for the different charging strategies.

account a number of other greenhouse gasses, and hence is not exactly equal to $\mathrm{CO}_{2}$, but differences are marginal and irrelevant for the discussion at hand in assessing $\mathrm{CO}_{2}$ emission reduction.) Based on these figures alone, we expect a substantial decrease in $\mathrm{CO}_{2}$ when we are able to increase use of wind generated energy. This expectation is verified by the results shown in Fig. $4 . \mathrm{CO}_{2}$ emissions are reduced by respectively $56 \%$ and $45 \%$ in the benchmark and smart charging scenario, compared to the business-as-usual scenario.

\section{CONCLUSION}

We presented a distributed algorithm for balancing supply from renewable energy sources and demand stemming from charging electric vehicles. Participants exchange energy consumption schedules and virtual price signals with a coordinator, and these are updated over multiple iterations, to achieve social welfare. This approach is scalable, and respects the privacy constraints: individual user preferences, exact energy requirements, and $\mathrm{EV}$ charging deadlines are kept private and not communicated to achieve coordination. Despite that limitation, we show that our approach closely approaches the all-knowing, optimal benchmark. This was quantitatively assessed in a simulation comprising 100 electric vehicles over the course of 4 weeks, during which renewable energy is supplied by a small scale wind turbine. Simulation results show that $68 \%$ of energy demand can be supplied by wind energy using the distributed algorithm, compared to $73 \%$ in an unrealistic best case scenario, and only $40 \%$ in a business-asusual scenario. Also, the increased usage of renewable energy sources, i.e. wind power, results in a $45 \%$ reduction of $\mathrm{CO}_{2}$ emissions, using our distributed algorithm.

Future work could explore a power system consisting of multiple balancing zones (i.e. multiple coordinators), each having their own renewable energy supply. Also, the current work only discussed charging while our approach can be extended to so-called vehicle-to-grid (V2G) services: vehicles could also provide temporary storage of energy, used for mobility purposes, or to be transferred to other vehicles at times of low availability of renewable energy.

\section{ACKNOWLEDGMENT}

K. Mets would like to thank the Agency for Innovation by Science and Technology in Flanders (IWT) for financial support through his Ph.D. grant. C. Develder is partially supported by the Research Foundation - Flanders (FWO - Vlaanderen) as a post-doctoral fellow. Work presented in this paper has been supported by the Flemish Government through the project SPARC (an IBBT ICON project).

\section{REFERENCES}

[1] C. of the European Communities, "20 20 by 2020 - Europe's climate change opportunity," 2008.

[2] P. Samadi, A. Mohsenian-Rad, R. Schober, V. Wong, and J. Jatskevich, "Optimal real-time pricing algorithm based on utility maximization for smart grid," in Proc. 1st IEEE Int. Conf. Smart Grid Communications (SmartGridComm2010), oct. 2010, pp. 415-420.

[3] P. Tarasak, "Optimal real-time pricing under load uncertainty based on utility maximization for smart grid," in Proc. 2nd IEEE Int. Conf. Smart Grid Communications (SmartGridComm2011), oct. 2011, pp. 321-326.

[4] N. Gatsis and G. B. Giannakis, "Residential load control: Distributed scheduling and convergence with lost ami messages," IEEE Trans. Smart Grid, p. 17, 2012, to appear.

[5] G. Webber, J. Warrington, S. Mariethoz, and M. Morari, "Communication limitations in iterative real time pricing for power systems," in Proc. 2nd IEEE Int. Conf. Smart Grid Communications (SmartGridComm2011), oct. 2011, pp. 445-450.

[6] S. Vandael, N. Boucké, T. Holvoet, K. De Craemer, and G. Deconinck, "Decentralized coordination of plug-in hybrid vehicles for imbalance reduction in a smart grid," in The 10th International Conference on Autonomous Agents and Multiagent Systems - Volume 2, ser. AAMAS '11. Richland, SC: International Foundation for Autonomous Agents and Multiagent Systems, 2011, pp. 803-810. [Online]. Available: http://dl.acm.org/citation.cfm?id=2031678.2031732

[7] K. Turitsyn, N. Sinitsyn, S. Backhaus, and M. Chertkov, "Robust broadcast-communication control of electric vehicle charging," in First IEEE International Conference on Smart Grid Communications, 2010.

[8] Elia, "Imbalance tariffs: compensation tariffs as an incentive for arps to maintain balance in their balancing perimeter." [Online]. Available: http://www.elia.be/en/products-and-services/ /media/files/ Elia/Products-and-services/ProductSheets/E-Evenwicht/E2_F_tarif_de_ compensation.ashx

[9] S. Boyd, L. Xiao, and A. Mutapcic, "Subgradient methods," Notes for EE392o, Stanford University, Autumn, 2003.

[10] J. Van Roy, N. Leemput, S. De Breucker, F. Geth, P. Tant, and J. Driesen, "An availability analysis and energy consumption model for a flemish fleet of electric vehicles," in European Electric Vehicle Congress (EEVC), Brussels, Belgium, October 26-28 2011, p. 12.

[11] W. V. Heddeghem, W. Vereecken, D. Colle, M. Pickavet, and P. Demeester, "Distributed computing for carbon footprint reduction by exploiting low-footprint energy availability," Future Generation Computer Systems, vol. 28, no. 2, pp. 405-414, 2012. 